\title{
SOME RESULTS ON ORDERED FILTERS OF IMPLICATIVE SEMIGROUPS
}

\author{
YOUNG BAE JUN
}

(Received 1 August 2000)

\begin{abstract}
We introduce a special set in an implicative semigroup. Using it, an equivalent condition of an ordered filter is stated. We prove that an ordered filter can be represented by the union of such sets.
\end{abstract}

2000 Mathematics Subject Classification. 20M12, 06F05, 06A06, 06A12.

1. Introduction. The notions of implicative semigroup and ordered filter were introduced by Chan and Shum [3]. The first is a generalization of implicative semilattice (see Nemitz [6] and Blyth [2]) and has a close relation with implication in mathematical logic and set theoretic difference (see Birkhoff [1] and Curry [4]). For the general development of implicative semilattice theory, the ordered filters play an important role which is shown by Nemitz [6]. To be motivated by this, Chan and Shum [3] established some elementary properties, and constructed quotient structure of implicative semigroups via ordered filters. Jun et al. [5] discussed ordered filters of implicative semigroups. To deeply study implicative semigroups, it is undoubtedly necessary to establish more complete theory of ordered filters for it. This paper continues investigations of implicative semigroups and of their ordered filters which were started in the general case by Chan and Shum [3]. We introduce a special set in an implicative semigroup. By using this set we give an equivalent condition of an ordered filter. We prove that an ordered filter can be represented by the union of such sets.

2. Preliminaries. We recall some definitions and results. By a negatively partially ordered semigroup (briefly, n.p.o. semigroup), we mean a set $S$ with a partial ordering " $\leq$ " and a binary operation "." such that for all $x, y, z \in S$, we have

(i) $(x \cdot y) \cdot z=x \cdot(y \cdot z)$,

(ii) $x \leq y$ implies $x \cdot z \leq y \cdot z$ and $z \cdot x \leq z \cdot y$,

(iii) $x \cdot y \leq x$ and $x \cdot y \leq y$.

An n.p.o. semigroup $(S ; \leq, \cdot)$ is said to be implicative if there is an additional binary operation $*: S \times S \rightarrow S$ such that for any elements $x, y, z$ of $S$,

(iv) $z \leq x * y$ if and only if $z \cdot x \leq y$.

The operation $*$ is called implication. From now on, an implicative n.p.o. semigroup is simply called an implicative semigroup.

An implicative semigroup $(S ; \leq, \cdot, *)$ is said to be commutative if it satisfies

(v) $x \cdot y=y \cdot x$, for all $x, y \in S$, that is, $(S, \cdot)$ is a commutative semigroup. 
In any implicative semigroup $(S ; \leq, \cdot, *), x * x=y * y$, and this element is the greatest in $S$; it will be denoted by 1 .

Some elementary properties of implicative semigroups are summarized by the following.

Proposition 2.1 (Chan and Shum [3, Theorem 1.4]). Let $S$ be an implicative semigroup. Then for every $x, y, z \in S$, the following statements hold:

(1) $x \leq 1, x * x=1, x=1 * x$,

(2) $x \leq y *(x \cdot y)$,

(3) $x \leq x * x^{2}$,

(4) $x \leq y * x$,

(5) if $x \leq y$, then $x * z \geq y * z$ and $z * x \leq z * y$,

(6) $x \leq y$ if and only if $x * y=1$,

(7) $x *(y * z)=(x \cdot y) * z$,

(8) if $S$ is commutative, then $x * y \leq(s \cdot x) *(s \cdot y)$ for all $s$ in $S$.

DEFINITION 2.2 (Chan and Shum [3, Definition 2.1]). Let $S$ be an implicative semigroup and let $F$ be a nonempty subset of $S$. Then $F$ is called an ordered filter of $S$ if

(F1) $x \cdot y \in F$ for every $x, y \in F$, that is, $F$ is a subsemigroup of $S$.

(F2) If $x \in F$ and $x \leq y$, then $y \in F$.

The following result gives an equivalent condition of an ordered filter.

Proposition 2.3 (Jun et al. [5, Proposition 2]). Suppose that $S$ is an implicative semigroup. Then a nonempty subset $F$ of $S$ is an ordered filter if and only if it satisfies the following conditions:

(F3) $1 \in F$,

(F4) $x * y \in F$ and $x \in F$ imply $y \in F$.

Now we note important elementary properties of a commutative implicative semigroup, which follows from (v), (1), and (7).

OBSERVATION. If $S$ is a commutative implicative semigroup, then for any $x, y, z \in S$,

(a) $x *(y * z)=y *(x * z)$.

(b) $y * z \leq(x * y) *(x * z)$.

(c) $x \leq(x * y) * y$.

3. Main results. In what follows let $S$ and $\mathbb{N}$ denote an implicative semigroup and the set of all positive integers, respectively, unless otherwise specified. For any elements $x$ and $y$ of $S$ and $n \in \mathbb{N}$, we use the notation $x^{n} * y$ instead of $x *(\cdots *(x *$ $(x * y)) \cdots)$ in which $x$ occurs $n$ times.

DeFinITION 3.1. For any $x, y \in S$ and $n \in \mathbb{N}$ define

$$
S_{n}(x, y):=\left\{z \in S \mid x^{n} *(y * z)=1\right\} .
$$

Obviously, $1, y \in S_{n}(x, y)$, for all $x, y \in S$ and $n \in \mathbb{N}$.

Proposition 3.2. If $S$ is commutative, then $x \in S_{n}(x, y)$ for all $x, y \in S$ and $n \in \mathbb{N}$. 
Proof. For any $x, y \in S$ and $n \in \mathbb{N}$ we have

$$
\begin{aligned}
x^{n} *(y * x) & =x^{n-1} *(x *(y * x)) \\
& =x^{n-1} *((x \cdot y) * x) \quad \text { by }(7) \\
& =x^{n-1} *((y \cdot x) * x) \quad \text { by }(\mathrm{v}) \\
& =x^{n-1} *(y *(x * x)) \quad \text { by }(7) \\
& =x^{n-1} *(y * 1) \quad \text { since } x * x=1 \\
& =x^{n-1} * 1 \quad \text { by }(1) \text { and }(6) \\
& =1,
\end{aligned}
$$

and so $x \in S_{n}(x, y)$.

Using (1) and (6) we have the following proposition.

Proposition 3.3. If $y \in S$ satisfies $y * z=1$ for all $z \in S$, then $S_{n}(x, y)=S=$ $S_{n}(y, x)$ for all $x \in S$ and $n \in \mathbb{N}$.

According to the following example, we know that there exists $k \in \mathbb{N}$ and $x, y \in S$ such that $S_{k}(x, y)$ is not an ordered filter of $S$.

EXAMPLE 3.4. Let $S:=\{1, a, b, c, d, 0\}$ be a set with Cayley tables (Tables 3.1 and 3.2) and Hasse diagram (Figure 3.1) as follows.

TABLE 3.1

\begin{tabular}{l|llllll}
\hline$\cdot$ & 1 & $a$ & $b$ & $c$ & $d$ & 0 \\
\hline 1 & 1 & $a$ & $b$ & $c$ & $d$ & 0 \\
$a$ & $a$ & $b$ & $b$ & $d$ & 0 & 0 \\
$b$ & $b$ & $b$ & $b$ & 0 & 0 & 0 \\
$c$ & $c$ & $d$ & 0 & $c$ & $d$ & 0 \\
$d$ & $d$ & 0 & 0 & $d$ & 0 & 0 \\
0 & 0 & 0 & 0 & 0 & 0 & 0 \\
\hline
\end{tabular}

TABLE 3.2

\begin{tabular}{l|llllll}
\hline$*$ & 1 & $a$ & $b$ & $c$ & $d$ & 0 \\
\hline 1 & 1 & $a$ & $b$ & $c$ & $d$ & 0 \\
$a$ & 1 & 1 & $a$ & $c$ & $c$ & $d$ \\
$b$ & 1 & 1 & 1 & $c$ & $c$ & $c$ \\
$c$ & 1 & $a$ & $b$ & 1 & $a$ & $b$ \\
$d$ & 1 & 1 & $a$ & 1 & 1 & $a$ \\
0 & 1 & 1 & 1 & 1 & 1 & 1 \\
\hline
\end{tabular}

It is easy to see that $(S ; \leq, \cdot, *)$ is an implicative semigroup. We know that the set $S_{2}(a, b)=\{1, a, b\}$ is an ordered filter of $S$, but $S_{2}(1, a)=\{1, a\}$ is not an ordered filter of $S$, because $a * b \in S_{2}(1, a)$ and $a \in S_{2}(1, a)$ but $b \notin S_{2}(1, a)$. 


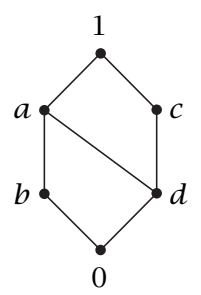

FIGURE 3.1

We give conditions for a set $S_{n}(a, b)$ to be an ordered filter.

THEOREM 3.5. Let $a \in S$ and $n \in \mathbb{N}$. Then $S_{n}(1, a)$ is an ordered filter of $S$ if and only if $a \leq z$ whenever $a \leq y$ and $a \leq y * z$, for all $y, z \in S$.

Proof. Suppose that $S_{n}(1, a)$ is an ordered filter of $S$. Let $y, z \in S$ be such that $a \leq$ $y$ and $a \leq y * z$. Then $1^{n} *(a * y)=a * y=1$ and $1^{n} *(a *(y * z))=a *(y * z)=1$. It follows that $y \in S_{n}(1, a)$ and $y * z \in S_{n}(1, a)$, which imply that $z \in S_{n}(1, a)$, that is, $1=1^{n} *(a * z)=a * z$. This shows that $a \leq z$. Conversely, assume that $a \leq y$ and $a \leq y * z$ imply that $a \leq z$ for all $y, z \in S$. Let $u, v \in S$ be such that $u * v \in S_{n}(1, a)$ and $u \in S_{n}(1, a)$. Then $1=1^{n} *(a *(u * v))=a *(u * v)$ and $1=1^{n} *(a * u)=a * u$, that is, $a \leq u * v$ and $a \leq u$. It follows that $a \leq v$ so that $1^{n} *(a * v)=a * v=1$, that is, $v \in S_{n}(1, a)$. Hence $S_{n}(1, a)$ is an ordered filter of $S$.

THEOREM 3.6. If $S$ satisfies the left self-distributive law under “*”, then $S_{n}(a, b)$ is an ordered filter of $S$ for all $a, b \in S$ and $n \in \mathbb{N}$.

Proof. Let $x, y \in S$ be such that $x * y \in S_{n}(a, b)$ and $x \in S_{n}(a, b)$. Using the self-distributivity and (1) it follows that

$$
\begin{aligned}
1 & =a^{n} *(b *(x * y))=a^{n} *((b * x) *(b * y)) \\
& =a^{n-1} *((a *(b * x)) *(a *(b * y))) \\
& \vdots \\
& =\left(a^{n} *(b * x)\right) *\left(a^{n} *(b * y)\right) \\
& =1 *\left(a^{n} *(b * y)\right)=a^{n} *(b * y)
\end{aligned}
$$

so that $y \in S_{n}(a, b)$. Therefore $S_{n}(a, b)$ is an ordered filter of $S$.

Using the set $S_{n}(a, b)$, we state a condition for a subset $F$ of $S$ to be an ordered filter of $S$.

THEOREM 3.7. Let $F$ be a nonempty subset of a commutative implicative semigroup $S$. Then $F$ is an ordered filter of $S$ if and only if $S_{n}(a, b) \subseteq F$ for every $a, b \in F$ and $n \in \mathbb{N}$.

Proof. Assume that $F$ is an ordered filter of $S$ and let $a, b \in F$ and $n \in \mathbb{N}$. If $x \in S_{n}(a, b)$, then $a^{n} *(b * x)=1 \in F$. Since $a, b \in F$, by using (F4) repeatedly we have $x \in F$. Hence $S_{n}(a, b) \subseteq F$. Conversely, suppose that $S_{n}(a, b) \subseteq F$ for every $a, b \in F$ and $n \in \mathbb{N}$. Note that $1 \in S_{n}(a, b) \subseteq F$. Let $x, y \in S$ be such that $x * y \in F$ and $x \in F$. 
Then

$$
\begin{aligned}
x^{n} *((x * y) * y) & =x^{n-1} *(x *((x * y) * y)) \\
& =x^{n-1} *((x * y) *(x * y)) \\
& =x^{n-1} * 1 \\
& =1,
\end{aligned}
$$

and so $y \in S_{n}(x, x * y) \subseteq F$. Thus $F$ is an ordered filter of $S$.

THEOREM 3.8. If $F$ is an ordered filter of a commutative implicative semigroup $S$, then $F=\cup_{a, b \in F} S_{n}(a, b)$ for every $n \in \mathbb{N}$.

Proof. Let $F$ be an ordered filter and let $x \in F$. Clearly $x \in S_{n}(x, 1)$, and so

$$
F \subseteq \underset{x \in F}{\cup} S_{n}(x, 1) \subseteq \underset{a, b \in F}{\cup} S_{n}(a, b) .
$$

Now let $y \in \cup_{a, b \in F} S_{n}(a, b)$. Then there exist $u, v \in F$ such that $y \in S_{n}(u, v)$. It follows from Theorem 3.7 that $y \in F$ so that $\cup_{a, b \in F} S_{n}(a, b) \subseteq F$. This completes the proof.

COROLLARY 3.9. If $F$ is an ordered filter of a commutative implicative semigroup $S$, then $F=\cup_{a \in F} S_{n}(a, 1)$ for every $n \in \mathbb{N}$.

\section{REFERENCES}

[1] G. Birkhoff, Lattice Theory, 3rd ed., American Mathematical Society Colloquium Publications, vol. 25, American Mathematical Society, Rhode Island, 1967. MR 37 \#2638. Zbl 153.02501.

[2] T. S. Blyth, Pseudo-residuals in semigroups, J. London Math. Soc. 40 (1965), 441-454. MR 31\#1211. Zbl 136.26903.

[3] M. W. Chan and K. P. Shum, Homomorphisms of implicative semigroups, Semigroup Forum 46 (1993), no. 1, 7-15. MR 93g\#20127. Zbl 776.06012.

[4] H. B. Curry, Foundations of Mathematical Logic, McGraw-Hill Book, New York, 1963. MR 26\#6036. Zbl 163.24209.

[5] Y. B. Jun, J. Meng, and X. L. Xin, On ordered filters of implicative semigroups, Semigroup Forum 54 (1997), no. 1, 75-82. MR 98a\#06022. Zbl 862.06005.

[6] W. C. Nemitz, Implicative semi-lattices, Trans. Amer. Math. Soc. 117 (1965), 128-142. MR 31\#1212. Zbl 128.24804.

Young Bae Jun: Department of Mathematics Education, Gyeongsang National UniVERSITY, JINJU 660-701, KOREA

E-mail address: ybjun@nongae.gsnu.ac. kr 


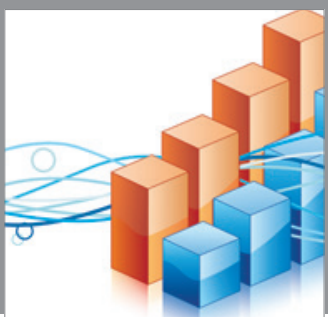

Advances in

Operations Research

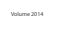

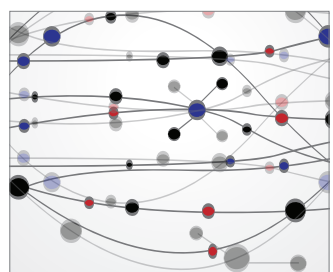

\section{The Scientific} World Journal
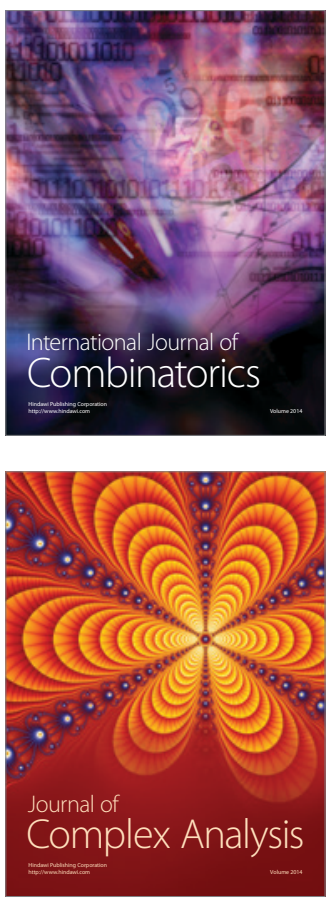

International Journal of

Mathematics and

Mathematical

Sciences
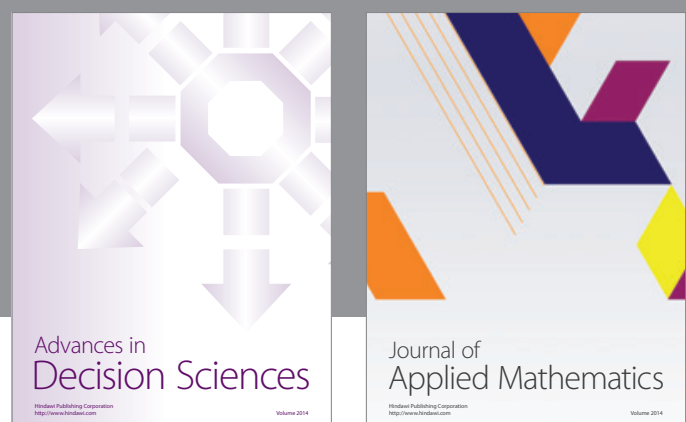

Journal of

Applied Mathematics
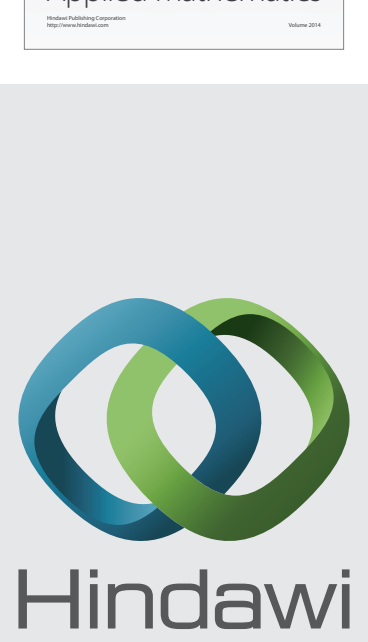

Submit your manuscripts at http://www.hindawi.com
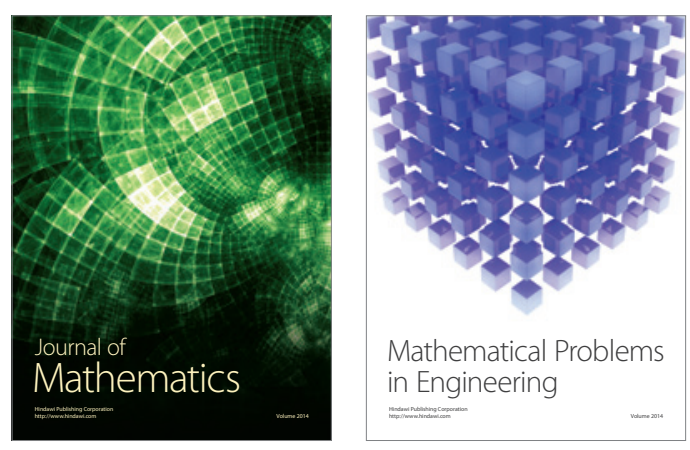

Mathematical Problems in Engineering
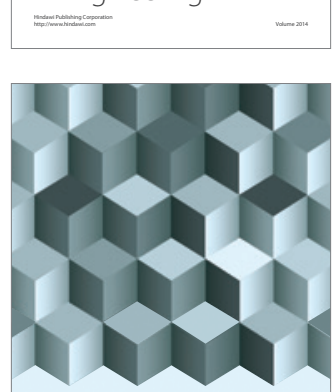

Journal of

Function Spaces
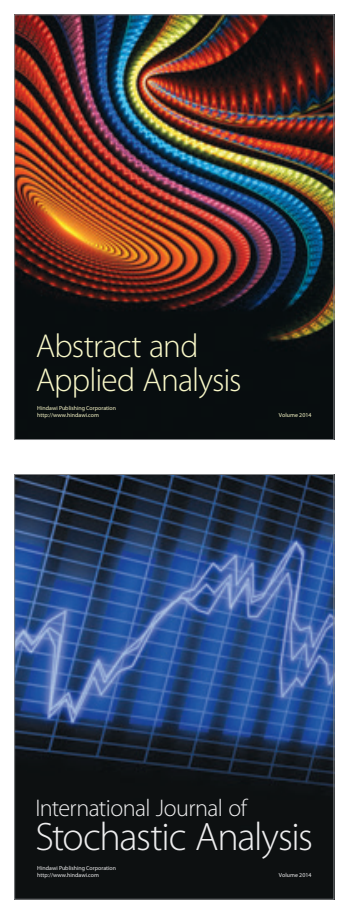

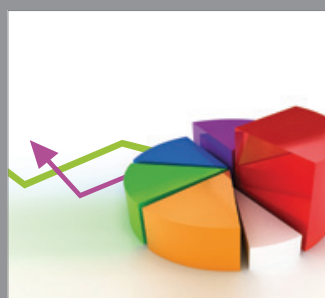

ournal of

Probability and Statistics

Promensencen
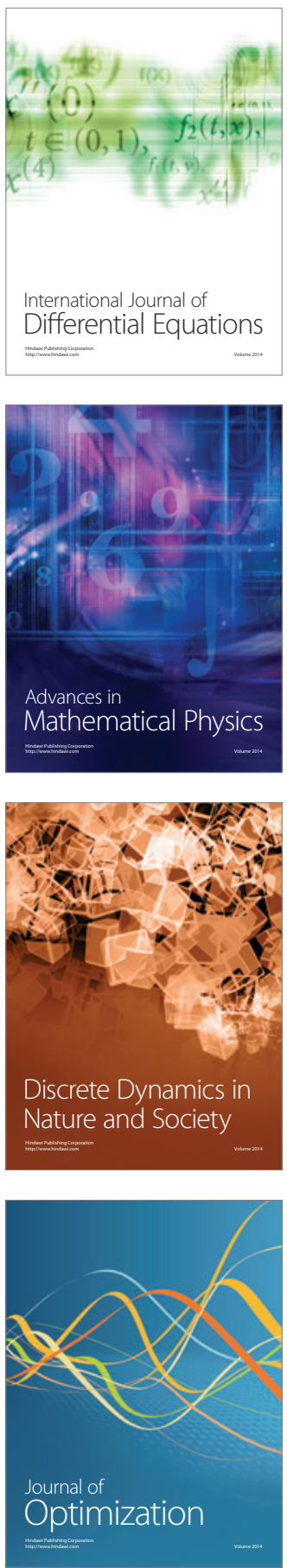